CAHIERS DE

NARRATOLOGIE

\section{Cahiers de Narratologie}

Analyse et théorie narratives

$36 \mid 2019$

Rhétorique et représentations de la culture mafieuse. Images, rituels, mythes et symboles

\title{
Il capo dei capi : une réprésentation polémique de Totò Riina
}

\section{Charlotte Moge}

\section{(2) OpenEdition \\ Journals}

Electronic version

URL: https://journals.openedition.org/narratologie/9834

DOI: $10.4000 /$ narratologie. 9834

ISSN: 1765-307X

Publisher

LIRCES

Electronic reference

Charlotte Moge, "/l capo dei capi : une réprésentation polémique de Totò Riina", Cahiers de Narratologie [Online], 36 | 2019, Online since 20 December 2019, connection on 21 September 2021. URL: http:// journals.openedition.org/narratologie/9834 ; DOl: https://doi.org/10.4000/narratologie.9834

This text was automatically generated on 21 September 2021.

Article L.111-1 du Code de la propriété intellectuelle. 


\title{
Il capo dei capi : une réprésentation polémique de Totò Riina
}

\author{
Charlotte Moge
}

1 Salvatore (alias Totò) Riina, né en 1930, débute sa carrière criminelle dans l'aprèsguerre. Originaire de Corleone, petit village de la province de Palerme, ce fils de paysan fait ses classes avec ses amis Calogero Bagarella et Bernardo (alias Binnu) Provenzano auprès de Luciano Liggio. Ensemble, ils assassinent le chef mafieux de Corleone en 1958 et une grande partie de ses hommes, prenant ainsi le pouvoir sur le territoire. Les Corléonais entrent ensuite dans les affaires de Palerme par la petite porte puis conquièrent la ville au terme d'une guerre terrible faisant un millier de morts au début des années $1980^{1}$. Dès lors, il en est fini de la gouvernance collégiale des grandes familles mafieuses, toutes décimées : Totò Riina devient le Capo dei capi (le parrain des parrains, le chef absolu) et gouverne Cosa nostra de manière unilatérale avec Bernardo Provenzano. En 1992, il commandite les massacres de Capaci et de via d'Amelio qui coûtent la vie aux juges Giovanni Falcone, Francesca Morvillo et Paolo Borsellino ainsi qu'à leurs escortes. En cavale depuis juillet 1969, il est finalement arrêté en janvier 1993 à Palerme ${ }^{2}$.

2 Attilio Bolzoni et Giuseppe D'Avanzo décident alors de retracer l'ascension criminelle des Corléonais et de Totò Riina en écrivant sa première biographie, Il capo dei capi. Vita e carriera criminale di Totò Riina qui est devenue depuis un ouvrage de référence. Spécialistes des questions mafieuses, ces deux journalistes ont opté pour un récit proche du roman, mais revendiquent dès l'introduction que tous les faits, dialogues et situations présentés sont « rigoureusement tirés des déclarations des témoins ou des actes judiciaires $»^{3}$. Ce statut hybride de la narration, où réel et fiction s'entremêlent, confère une force certaine à la reconstruction proposée et l'histoire est adaptée en fiction télévisée pour Canale 5 en $2007^{4}$. Outre le sérieux de la biographie originale, la participation de Claudio Fava $^{5}$ à l'écriture du scénario garantit a priori une représentation désacralisante de la figure de Totò Riina. Pourtant, si la biographie véhicule l'image d'un chef mafieux impitoyable et sanguinaire, fidèle à son surnom « la bête ", la série atténue cet aspect, tout en racontant pratiquement les mêmes faits et en 
reprenant parfois les mêmes dialogues. Il convient dès lors de s'interroger sur les ressorts de cette représentation car l'image du personnage forge également sa mémoire auprès du public, a fortiori dans le cas d'une série télévisée qui, comme le Capo dei capi, rencontre un large succès, y compris à l'étranger. Ce pouvoir de façonner la mémoire nous impose d'étudier non seulement la représentation en soi et le message véhiculé mais également la réception de la série lors de sa diffusion. Nous analyserons donc la sédimentation des différentes facettes du personnage de Totò Riina afin de comprendre pourquoi il s'agit d'une représentation problématique et mythifiante, dont le succès a déclenché de vives polémiques.

\section{La sédimentation des différentes facettes du personnage}

\section{Le « viddano » de Corleone}

Comme la biographie, la série commence le récit de la vie de Totò Riina par un événement tragique déterminant. En septembre 1943, son jeune frère Francesco et son père meurent en manipulant une bombe alliée dont ils voulaient extraire la poudre pour la revendre. "Ce n'est pas la bombe qui a tué mon père, c'est la faim ${ }^{6}$ " dit le personnage de Riina dès le début. Sortir de la misère devient le moteur de l'ambition dévorante du jeune Totò qui ne fera qu'une bouchée de ses ennemis. Ainsi, le début des manœuvres pour s'émanciper de la tutelle des Palermitains est symboliquement représenté par un gros plan sur la bouche de Riina qui mange un quartier d'orange. De même, au moment de lancer l'offensive contre les familles palermitaines, il fait de cet appétit le ciment de la cosca, la famille mafieuse des Corléonais : « Notre force, c'est la faim ! À Palerme, ils ne savent même pas ce que c'est. Mais moi je l'ai ressentie. Et une fois que tu l'as ressentie, tu la gardes chevillée au corps toute ta vie $»^{7}$.

Attilio Bolzoni et Giuseppe D'Avanzo ont estimé au terme de leur biographie que Riina a tiré sa force de "chef de meute ${ }^{8} »$ de sa condition de paysan, au point d'en faire une marque de fabrique :

Les Corléonais descendirent de leurs collines et entrèrent timidement en ville avec leurs vêtements en tissu noir ou en velours à larges côtes, leurs chaussures crottées de boue, leur béret sur la tête. Ils avaient le visage hâlé par le soleil et les mains calleuses. Ils étaient humbles, respectueux, parfois même serviles. Les vice-rois ${ }^{9}$ les regardaient et se payaient leur tête, ceux qui venaient de la campagne étaient des péquenauds ${ }^{10}$.

La série est fidèle à cette clé d'interprétation et montre bien que cette image de viddani (péquenauds) a été le véritable cheval de Troie des Corléonais pour infiltrer les familles palermitaines sans que celles-ci ne prennent la mesure du danger. La différence entre les mafieux de la ville et ceux de la province s'incarne dans les tenues vestimentaires car le style campagnard des Corléonais est loin de l'élégance des Palermitains. Au fil des épisodes, plus il assoit son statut de chef, plus Totò Riina - incarné par Claudio Gioè prend de l'embonpoint et se mimétise en véritable paysan. À partir de l'épisode 3, on le voit jardiner, ramasser des oranges, prendre soin des tomates tandis qu'au début du dernier épisode, il va nourrir ses poules en intimant à Ignazio Salvo ${ }^{11}$ - qui lui promet que le verdict du maxi-procès ${ }^{12}$ sera annulé en Cassation - de le suivre dans le poulailler

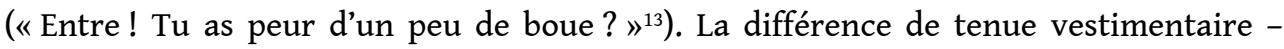
Riina revêt des bottes en caoutchouc et un gilet en grosse laine tandis que Salvo est en 
costume et chaussures de ville - montre un Riina qui n'a pas peur de se salir les mains et donc de mettre ses menaces à exécution si ses appuis politiques ne maintiennent pas leurs promesses. Cette image rustique est renforcée par la langue, un italien teinté d'expressions dialectales souvent utilisées pour donner des ordres ou pour nier son statut de chef mafieux et se présenter comme un simple paysan lorsqu'il est arrêté : "Ma io un viddano sono. Uno con i peri incritati ( (" Mais moi je suis un péquenaud qui a les pieds crottés $»^{14}$.

\section{Le stratège}

6 Cette image de campagnard humble et servile est évidemment un leurre et fait partie de la stratégie mise en place par Totò Riina pour s'emparer de Cosa nostra. La série met bien en évidence ce double jeu et fait remonter sa passion pour la tactique à sa première incarcération, lorsque Gaspare Mutolo ${ }^{15}$ lui apprend à jouer aux dames. Les parties de dames sont la métaphore des rapports de forces qui se modifient au sein de Cosa nostra. Alors qu'il joue avec Provenzano, Riina le met en garde : «Binnu, avant de bouger ton pion, tu dois penser à ce que moi j'ai en tête. Si tu ne penses qu'à ton jeu, je te nique. Comme les Palermitains ${ }^{16} »$. Les épisodes 2,3 et 4 , consacrés à la prise de pouvoir des Corléonais sur les familles palermitaines, construisent l'image d'un Riina rusé et habile. Sa stratégie de conquête consiste à accumuler les soutiens des hommes de main, les picciotti de toutes les cosche de Palerme afin de recueillir des informations pour semer ensuite la zizanie entre les chefs. Riina se montre donc proche des plus petits, en les soudoyant ou en leur promettant des postes de commandement ( Ceux qui ont trimé n'oublient pas ceux qui sont en bas de l'échelle $\left.\aleph^{17}\right)$. Le recours à une métaphore botanique pour expliquer sa stratégie à Provenzano ( La graine a germé. Et si tu ne l'arroses pas, elle va mourir. Nous on va la faire grandir et ensuite on va prendre toute la plante $»^{18}$ ) montre que c'est grâce à la conjonction de ces deux dimensions que Riina, le campagnard-stratège, réussit à berner les Palermitains.

7 Les scènes des summit, les réunions des principaux chefs mafieux, contribuent activement à la construction de cette dimension du personnage. Au début, Riina écoute à l'écart et n'intervient que pour se montrer serviable et dévoué. Dès que les Corléonais sont mis en cause ${ }^{19}$, il nie leur implication en dénonçant l'action de familles "qui viennent de l'extérieur ${ }^{20}$. Dans l'épisode 3, Giuseppe Di Cristina, chef mafieux de Riesi, avertit les boss palermitains qu'il a «découvert» que les familles des provinces estiment que les responsables sont les Corléonais. Riina prend alors la parole :

Je respecte Giuseppe Di Cristina, je suis sûr qu'il parle en toute bonne foi. On peut découvrir beaucoup de choses. Christophe Colomb, par exemple, a découvert l'Amérique. Elle existe, elle est là, c'est un fait. Mais l'Amérique c'est une chose, la rumeur, ça en est une autre. Si quelqu'un ne découvre que des on-dit et des rumeurs, dites-moi de quel genre de découverte s'agit-il? Disons que je me promène et qu'on me dit que Di Cristina est énervé contre moi parce que j'ai récupéré des appels d'offres de Cavataio qu'il pensait récupérer pour lui. Je peux pas venir vous voir en vous disant que j'ai découvert que Di Cristina dit ce qu'il dit parce qu'il veut ma perte et prendre ces appels d'offres. Ça, je ne peux pas vous le dire, parce que ce n'est pas une découverte. Ce ne sont que des on-dit et des rumeurs. Des on-dit et des rumeurs qui peuvent nous amener à faire une guerre inutile entre amis. Les amis en revanche doivent être tranquilles et avoir confiance $^{21}$. 
l'image du personnage car on voit apparâtre un Riina sournois et beau parleur, qui arrive à retourner l'assemblée. Son discours est si convainquant que Tommaso Buscetta propose que les Corléonais intègrent la Cupola. Riina veut ensuite embrasser Di Cristina : «On exagère tous parfois avec les mots. Mais qu'est-ce que c'est, les mots ? De l'air. Et faire de l'air ça veut dire qu'on est vivant. Et ça c'est bien. »Cette magnanimité feinte lui permet de donner un véritable baiser de la mort à Di Cristina, qu'il fait tuer peu après. La représentation filmique montre le stratège décomplexé et impitoyable qu'était Riina, qualifié de «serpent" par le repenti Antonino Calderone pour avoir prononcé un éloge funèbre à la mémoire de son frère après l'avoir fait assassiner ${ }^{22}$.

9 La série insiste également sur les effets délétères de la stratégie de Riina sur les Palermitains une fois qu'ils comprennent qu'ils ont été bernés. Stefano Bontate, initialement calme et pondéré, invoquant toujours le respect des règles, perd son sangfroid et, aveuglé par la haine, veut tuer Riina de ses propres mains, mais celui-ci est introuvable. Buscetta le met en garde avant de s'exiler au Brésil : « Il a pris possession de ton âme et d'ici peu il prendra ton corps $»^{23}$. Un des picciotti soudoyés rend compte de la situation à Riina : « les Palermitains deviennent fous, ils vous cherchent comme le chat traque la souris ». Riina lève les yeux, menaçant : « et la souris, ce serait moi ? Tout ce travail pour être la souris ? [...] La souris en a marre, et elle veut devenir le chat ${ }^{24}$. Par cette métaphore, Riina lance les hostilités contre les Palermitains en faisant exécuter Bontate, puis Inzerillo. L'épisode se conclut sur la venue de Johnny Gambino, boss des familles new-yorkaises, qui rencontre Totò Riina et reconnaît son statut de chef absolu.

\section{Du capo famiglia au capo dei capi}

10 L'hégémonie du personnage de Riina s'affirme tout au long de la représentation de sa carrière criminelle. Dès le premier épisode, le jeune Totò commence à s'imposer en partageant le butin, à l'époque constitué de viande, de fromage, de farine et d'un peu d'argent. À l'épisode suivant, Liggio avait promis à Riina que Luciano Maino ${ }^{25}$, un de leurs complices qui avait décidé de collaborer avec la justice, ne témoignerait pas au procès de Bari. Après son témoignage devant les juges, Riina prend le leadership sur Liggio en lui disant : « Maintenant, on fait à ma façon $»^{26}$. Les juges reçoivent alors une lettre anonyme de menaces, dont le contenu est lu par la voix off de Riina, et le procès se conclut par un acquittement général. Dans le troisième épisode, Riina explique à Provenzano qu'ils doivent s'émanciper de l'autorité de Liggio et organiser des enlèvements ${ }^{27}$. Même s'il réside Milan, Liggio reste le chef des Corléonais et est membre de la Cupola de Cosa nostra, mais Riina le remplace souvent. La concurrence entre les deux hommes augmente et Liggio est arrêté en 1974. Les chefs palermitains soupçonnent d'emblée Riina de s'être débarrassé de Liggio en dénonçant sa présence à Milan. Dès lors, il devient le véritable capo famiglia des Corléonais.

11 Nous l'avons $\mathrm{vu}$, la consolidation du statut de chef de Riina s'accompagne du renforcement de son image de paysan. Ainsi, les nombreuses séquences où il cultive la terre sont fonctionnelles à l'économie du récit car les montages parallèles montrent ses picciotti exécutant ses ordres. La représentation déséquilibrée du binôme RiinaProvenzano, avec un Provenzano qui doute de la réussite du plan élaboré par Riina ou qui semble ne pas en comprendre d'emblée la portée, renforce la suprématie de Riina et

Cahiers de Narratologie, 36 | 2019 
relègue Provenzano au rôle d'homme de main puis de conseiller ${ }^{28}$. Après la mattanza ${ }^{29}$, Riina affirme son statut de chef absolu auprès de John Gambino, justifiant l'élimination des autres familles : " Il y avait trop de têtes qui commandaient. Maintenant, il n'y en a plus qu'une $"^{30}$. L'épisode suivant s'ouvre sur une scène où Riina s'adresse à ses hommes et revendique ce nouveau mode de fonctionnement dictatorial :

Voilà ce qu'est la démocratie, mes amis. Des bavardages, des bavardages qui font perdre du temps et de l'argent. [...] Vous ne savez pas que Cosa nostra aussi devenait une démocratie ? Et moi je me suis dit: «Totò, là il faut régler cette situation ». Parce que certaines affaires compliquées ne peuvent pas se régler en démocratie. Quand il faut prendre une décision, il faut quelqu'un qui soit capable de la prendre pour tout le monde. [...] Il faut qu'on change nos habitudes. Le temps des bavardages est terminé. Et celui qui ne veut pas le comprendre devient mon ennemi. Et donc il meurt ${ }^{31}$.

La mise en scène vient appuyer le discours puisque Riina est le seul debout et il se déplace au milieu des autres mafieux qui, eux, sont assis et immobiles. L'alternance des plans d'ensemble sur l'auditoire et des gros plans sur Riina vise à montrer l'acceptation du discours et donc de la suprématie du chef. Le plan final de Riina en contre-plongée assoit définitivement sa domination absolue sur cosa nostra, basée sur la terreur.

13 La série insiste sur la transformation de Riina en leader tyrannique de plus en plus solitaire et nerveux, comme en témoignent ses brûlures d'estomac. Dans les deux derniers épisodes, il devient irascible et paranoïaque, soupçonnant même son épouse d'essayer de lui soutirer des informations sur ses intentions pour le compte de son frère ou de Provenzano. L'isolement du chef est renforcé par la représentation de la rupture progressive avec Provenzano, qui le met en garde contre les avancées des forces de l'ordre et contre sa gestion totalitaire de Cosa nostra: "Tu changes. On se connait depuis une éternité et je te dis que tu n'écoutes plus $»^{32}$. Les scènes de tension entre les deux hommes augmentent, notamment après la confirmation du verdict du maxiprocès par la Cour de Cassation ${ }^{33}$. Riina commence alors à se méfier de Provenzano, comme en témoigne cet échange avec son chauffeur Balduccio Di Maggio :

TR : Je te semble différent?

BDM : Non.

TR : Et Binnu?

BDM : Je le vois un peu plus nerveux.

TR : J'ai confiance en lui, tu le sais. On a grandi ensemble. Même pain, même faim.

C'est juste que de temps en temps j'ai l'impression qu'il veut me doubler.

$\mathrm{BDM}$ : Binnu est fidèle, $z^{34}$ Totò.

TR : Ce sont les chiens qui sont fidèles. Je me fais vieux et avec la vieillesse, je me fais du mauvais sang ${ }^{35}$.

Ces doutes sur la loyauté de Provenzano vont pousser Riina à s'enferrer dans une stratégie de la violence et à décréter les assassinats des juges Falcone ${ }^{36}$ et Borsellino. Cet isolement décisionnel, représenté dans une scène où Riina joue désormais seul aux dames, est le fruit d'un désaccord stratégique entre les deux hommes ${ }^{37}$. Lorsque des carabiniers tentent de négocier l'arrêt des massacres par l'intermédiaire de Vito Ciancimino $^{38}$, pendant la période dite la trattativa (la négociation), Riina, en proie à un délire de toute-puissance, s'adresse à Provenzano en ces termes : « Ils doivent négocier avec moi. Ici, l'État c'est moi $»^{39}$. Il s'agit de la dernière scène où ils apparaissent ensemble. La violence de l'échange ainsi que l'alternance des gros plans sur les regards de défi que les deux hommes se lancent mutuellement montrent que la rupture est 
consommée. Le Capo dei capi, désormais totalement seul aux commandes, apparaît inéluctablement sur le déclin et de fait, il sera arrêté peu après.

\section{Une représentation mythifiante et problématique}

\section{Irréalisme de l'antimafia}

15 Si la représentation de la carrière criminelle de Totò Riina est assez réaliste, il n'en va pas de même pour les activités de l'antimafia. Afin d'équilibrer la représentation et de laisser une place à l'antimafia comme c'est le cas dans la biographie, les scénaristes de la série ont choisi d'inventer un antagoniste à Riina, Biagio Schirò. Ami d'enfance de Riina, Provenzano et Bagarella, Schirò commence à travailler lui aussi pour Luciano Liggio. Il quitte le groupe lorsqu'il comprend que ses amis sont responsables de la mort de Placido Rizzotto, le syndicaliste tué en 1948, puis fait des études pour devenir policier. Il consacre ensuite sa vie à traquer Riina. Le personnage de Schirò est censé incarner tous ceux qui ont dédié leur vie à lutter contre la mafia mais cette personnification de l'antimafia chorale se révèle paradoxale et problématique à bien des effets.

L'intention de montrer la cohérence de l'action antimafia menée sur plusieurs décennies par plusieurs acteurs qui ont tous connu un destin funeste ne fonctionne pas car presque toutes les intuitions novatrices sont attribuées à Schirò (c'est par exemple lui qui suggère à Giovanni Falcone d'interroger Tommaso Buscetta), ce qui rend la représentation de l'antimafia complètement irréelle. Sa connaissance parfaite des Corléonais lui vaut d'être qualifié de "phénomène » et d'" archive vivante " par les commissaires Ninni Cassarà et Beppe Montana ${ }^{40}$. Schirò est un être hors norme qui éclipse les autres acteurs de la lutte contre la mafia. Sa crédibilité d'enquêteur qui se méfie de tout et de tous est construite au détriment des véritables protagonistes de l'antimafia, comme en témoignent les entrevues avec le procureur Gaetano Costa ${ }^{41}$ ou la rencontre avec Giovanni Falcone, dont il met en doute des compétences :

BS : Vous êtes l'un de ces gentlemen qui ont refusé de signer les mandats d'arrêt ${ }^{42}$ ?

GF : Je travaille sur le procès Bontate.

BS : C'est bizarre, je ne vous ai jamais vu.

GF : Je viens d'être transféré.

BS : Nous voilà dans de beaux draps, on confie l'affaire au dernier arrivée ${ }^{43}$.

Schirò incarne le pessimisme de la lutte contre la mafia, mais sa lucidité et son insatisfaction permanente donnent à voir un état d'esprit opposé à celui des acteurs de l'époque, dont tous les témoignages soulignent combien ils croyaient en leur combat et étaient conscients que chaque petite avancée était une victoire ${ }^{44}$. De plus, la création d'une personnalité hégémonique de l'antimafia ternit l'image des véritables acteurs de la lutte contre la mafia : leurs choix semblent moins courageux car suggérés par Schirò (c'est lui qui pousse Gaetano Costa à signer des mandats d'arrêts), et ils apparaissent tantôt soumis à la hiérarchie (dans le cas de Boris Giuliano ${ }^{45}$ ), tantôt utopistes (dans le cas de Giovanni Falcone, qui essaie de lui « redonner le sourire » en lui montrant la liste de tous les mandats d'arrêts émis ${ }^{46}$ ). Schirò paye cher son engagement puisqu'il voit ses collègues mourir les uns après les autres. Au dernier épisode, il se définit comme «le gardien d'un cimetière » et refuse que son fils Antonio suive son chemin car «c'est une vie de merde ${ }^{47} »$. Cette affirmation semble donner raison à Riina puisqu'elle fait écho à ses propos lorsqu'il décide d'épargner Schirò à la fin de l'épisode $3:$ "Toi, pour moi, tu 
es comme la télévision. Je te regarde et, en voyant ta vie, je me dis que j'ai bien fait de choisir la mienne ${ }^{48} »$. Une telle représentation de l'antimafia s'avère risquée car elle inverse les perspectives : le modèle vainqueur est celui de Riina.

Pourtant, dans les premiers épisodes, la création d'un antagoniste équilibre en effet le récit. Les montages parallèles opposent un modèle positif, celui de la vie, à un modèle négatif, celui de la violence et de la mort: tandis que le fils de Biagio voit le jour, Calogero Bagarella meurt dans le massacre de viale Lazio $^{49}$. Toutefois, cette dynamique s'inverse rapidement : alors que la femme de Riina accouche de leur troisième enfant, celle de Biagio fait une fausse couche à cause du stress et décide ensuite de quitter Palerme avec son fils pour aller vivre à Rome. Ainsi, pendant que Totò Riina vit une cavale heureuse avec femme et enfants, Biagio Schirò est seul à Palerme. La tentative de personnifier le martyrologe de la lutte contre la mafia fournit certes la stabilité d'une figure positive mais la fiction se conclut sur la représentation d'un homme fatigué et boiteux (il avait été blessé par les Corléonais à la fin de l'épisode 5), qui incarne l'image d'un État considérablement affaibli par la lutte contre la mafia. Le face-à-face entre les deux hommes qui ouvre et clôt la série après l'arrestation de Riina laisse un goût amer. La représentation irréelle de l'antimafia ne permet donc pas de prendre la mesure de la victoire que constitue l'arrestation du capo dei capi et contribue ainsi à mythifier la figure de Riina.

\section{Mythification de Riina}

19 L'histoire de l'ascension criminelle de Riina, suivant la sédimentation des figures que nous avons analysée plus haut, est le récit d'une success story qui héroïse d'abord, puis mythifie la figure du chef mafieux. L'enfant de la misère qui devient le chef absolu de la mafia véhicule le modèle problématique de la carrière mafieuse comme ascenseur social. Gagner seul contre tous fait de lui un héros tandis que ses qualités de chef et de stratège en font une figure mythique. Claudio Gioè, qui interprète Riina, semble conscient de ce risque :

$\mathrm{Au}$ début, en expliquant que Riina a souffert de la faim pendant son enfance, il peut $\mathrm{y}$ avoir le risque que le personnage plaise. En tant que Siciliens, on sait combien la mafia peut être sournoise et charmante. Dans les épisodes suivants, sa férocité et celle des autres émerge ${ }^{50}$.

Toutefois, cette férocité est plus sous-entendue que représentée explicitement. Certes, au début on le voit exécuter des homicides, mais la représentation ne suffit pas à restituer l'image d'une bête sanguinaire ${ }^{51}$. La focalisation sur la stratégie mise en place pour prendre le pouvoir au sein de Cosa nostra porte le spectateur à considérer les meurtres comme des étapes inévitables de son ascension et comme l'expression d'un plan issu d'un esprit brillant, posant ainsi un double problème éthique. Le personnage de Riina n'éprouve évidemment aucun remords, mais ce manque d'empathie apparaît comme une qualité nécessaire pour s'imposer. Même si les assassinats représentés sont nombreux, la série ne peut pas être définie comme violente, le sang ne coulant pas à flots.

21 À partir de l'épisode 3, Riina ne se salit plus les mains lui-même et commandite les assassinats en une phrase. Le plus souvent, ses ordres sont insérés dans des discussions ou des situations du quotidien. En créant le contraste, le parti pris des auteurs est de montrer la cruauté du personnage pour qui cette violence est banale. Il ordonne par 
exemple l'homicide de Pio La Torre en jouant au foot avec son fill ${ }^{52}$ ou encore la torture d'un mafieux alors qu'il emmène ses enfants acheter des jouets. Ensuite, tandis qu'il monte une balançoire avec Provenzano, il commandite les assassinats des enfants de Tommaso Buscetta : "Faites-lui savoir que son sang ne vieillira pas " ${ }^{53}$. Les montages parallèles le montrant en famille pendant que les picciotti exécutent ses ordres servent à insister sur son inhumanité. Cette séquence où s'alternent les plans montrant ses enfants qui s'amusent sur la balançoire et ceux représentant l'enlèvement, l'assassinat et la dissolution dans l'acide des enfants de Buscetta est certainement la plus suggestive. La figure négative du chef mafieux se télescope ainsi avec celle, positive, du père de famille. Cependant, malgré l'horreur de la situation, le fait qu'il n'agisse pas luimême et qu'il soit longuement représenté dans son quotidien de père aimant tout au long de la série déséquilibre la représentation. Sa monstruosité est en quelque sorte atténuée par son humanité. La complexité de sa personnalité, revendiquée par les auteurs ( Il est impensable que les méchants ne soient que méchants » ${ }^{54}$ ), n'entache pas le mythe mais, bien au contraire, l'alimente.

\section{La série en question?}

Dès lors, c'est bien le format utilisé qui pose question. La série ne favorise-t-elle pas encore davantage la mythification? Composée de six épisodes d'une heure et demie chacun, soit une durée totale de plus de neuf heures, la fiction est très longue. Cette longueur est d'autant plus problématique que les diverses productions cinématographiques réalisées précédemment sur les figures de l'antimafia sont soit des film $s^{55}$ soit des mini-séries en deux épisodes ${ }^{56}$. Ce déséquilibre participe là aussi de la mythification de la figure de Riina, à qui l'on consacré trois fois plus de temps. Pietro Grasso, procureur national antimafia lors de la diffusion de la série sur Canale 5, critique la fiction justement sur ce point :

Si elle a un défaut, c'est d'avoir été diffusée en épisodes et dans quelques-uns le côté fascinant du personnage pourrait ressortir. $\mathrm{Si}$, en revanche, elle avait été ramassée en deux heures, on serait arrivé tout de suite à la morale qui, selon moi, est plus éducative que tout le reste de la fiction ${ }^{57}$.

La division en épisodes crée naturellement un suspense et une évolution du personnage auquel, malgré ses actions criminelles, le spectateur s'attache. L'utilisation des surnoms dans les dialogues crée un effet de proximité et de familiarité auprès du public. La représentation du quotidien du bon père de famille contribue à créer une identification tandis que la succession des différents acteurs de l'antimafia (qu'il fait éliminer) ainsi que l'isolement de Schirò renforcent son prestige criminel, donnant l'impression qu'il est toujours vainqueur. Le destin des criminels étant généralement la mort ou la prison, son arrestation n'est que la conclusion logique de son parcours. La morale, certes positive, pèse ainsi bien moins lourd que le modèle véhiculé, ce qui n'a pas manqué de déclencher de vives polémiques.

\section{Succès et polémiques}

\section{L'accueil du public}

Lors de sa diffusion, la fiction connait d'emblée un franc succès. Le premier épisode est suivi par environ 7146000 téléspectateurs et arrive en tête des audiences (plus de 
$\left.27{ }^{58}\right)$. Le succès ne se dément pas jusqu'au dernier épisode, qui recueille près de 8 millions de téléspectateurs et près de $28 \%$ des parts d'audience, juste derrière la retransmission en direct du spectacle de Roberto Benigni consacré à la lecture de la Divine Comédie ${ }^{59}$. La fiction a fait couler beaucoup d'encre. De nombreuses voix, dont celle de Clemente Mastella alors ministre de la Justice, dénoncent la mythification de la figure de Riina et mettent en garde contre les dangers pour les jeunes générations de s'identifier au personnage. Ces critiques véhiculées par la presse sont discutées dans des forums sur internet. Le blog palermitain Rosalio.it ouvre une section de débat «Il capo dei capi : pour ou contre ? $»^{60}$, où les Palermitains postent 95 commentaires : une majorité réfute le risque d'émulation et apprécie la série, qui a le grand mérite de montrer les dynamiques criminelles internes à la mafia. Tous s'accordent pour dire que représenter la mafia est un moyen de briser l'omerta, mais plusieurs dénoncent la représentation irréaliste de l'antimafia (en particulier de Boris Giuliano) ${ }^{61}$ et l'invention de la figure de Biagio Schirò ainsi que le choix du titre qui participe de l'héroïsation du personnage de Riina, jugeant que "La belva », en référence à son surnom, aurait été plus approprié.

Au-delà du personnage de Totò Riina, la représentation de la mafia proposée dans Il capo dei capi génère la même fascination qui est à l'origine du succès des films de gangsters italo-américains. Le spectateur cinéphile retrouve quelques analogies avec des scènes cultes de films d'anthologie comme Le Parrain de Francis Ford Coppola (1972) et Les Affranchis de Martin Scorsese (1990) ${ }^{62}$. La représentation d'un Riina à la fois père de famille et chef mafieux rappelle également Don Vito Corleone. Le film de Coppola, tiré du roman éponyme de Mario Puzo publié en 1969, est sorti bien avant que les Corléonais ne prennent le pouvoir sur les autres familles mafieuses, mais le nom du personnage principal a donné une résonnance internationale au village de Corleone. Cette célébrité a certainement conduit le choix des distributeurs français à changer le titre de la série en "Corleone ». L'association du mythe cinématographique et du récit de la success story de Totò Riina ont permis à la version française de la série de connaître un très large succès ${ }^{63}$, en particulier dans les milieux de la petite et moyenne délinquance. Nous avons personnellement constaté la popularité de la série auprès de jeunes des quartiers difficiles ayant maille à partir avec la justice et avons également eu connaissance du succès de la série dans une prison française, où les détenus suivant des cours d'italien souhaitaient continuellement parler de Totò Riina. Ces expériences nous ont permis de vérifier le pouvoir de mythification de la série puisque pour ces jeunes, Totò Riina est un modèle de ruse et de réussite.

\section{L'intérêt des mafieux}

Il capo dei capi a également suscité l'intérêt de Totò Riina. Au lendemain de la diffusion du premier épisode, un article de La Repubblica consacré aux audiences record et au boss devant l'écran dans sa cellule, explique que Riina «a renoncé à son habitude maniaque d'aller se coucher à dix heures » :

Pendant deux heures, il est resté immobile devant sa vie qui défilait dans le petit écran [...]. Riina est resté impassible quand il s'est revu enfant dans le Corleone de l'après-guerre. [...] Lui, le vrai parrain, n'a montré aucune émotion pour la scène de sa première rencontre avec Luciano Liggio ${ }^{64}$.

L'attention que le journaliste porte aux habitudes et aux réactions de Riina - qui lui ont certainement été rapportées par les gardiens - montre combien la réception de la série 
par le premier intéressé suscite la curiosité. La diffusion de la série est un tel événement médiatique que sa réception le devient aussi. Riina n'ayant jamais montré une quelconque émotion lors de ses procès - si ce n'est du mépris envers les mafieux repentis - il eût été étrange qu'il le fasse en regardant la télévision, se sachant soumis à un contrôle très strict. Mais le fait même de scruter ses réactions témoigne de l'envie de percer le mystère du personnage. La représentation de la mafia peut fasciner, mais la réception de cette représentation intrigue encore plus. L'auteur de l'article, spécialiste de la mafia, s'est également informé sur la réaction de Bernardo Provenzano, qui s'avère être opposée à celle de Riina :

Pour un soir, Riina a renoncé à sa passion pour les émissions de sport. Provenzano, en revanche, a suivi les dernières nouvelles des journaux télévisés et à neuf heures, ponctuel, il s'est couché65.

Signaler le désintérêt de Provenzano sert à souligner la curiosité de Riina qui, selon son avocat, s'inquiétait de voir « comment seraient racontés (sa) femme et surtout (ses) enfants. L'affaire judiciaire est une chose, la vie privée en est une autre $»^{66}$. Riina s'est ensuite plaint auprès de son avocat non pas du récit de ses activités criminelles - qu'il a pourtant toujours niées - mais de la représentation de sa femme Ninetta («Qu'est-ce que ma femme a à voir avec tous ces mensonges ? $\left.{ }^{67}\right)$.

Il est impossible de mesurer l'impact de la représentation de Il Capo dei capi sans réaliser une enquête approfondie, mais on peut signaler qu'elle suscite l'intérêt de quelques mafieux comme Michele Catalano qui, comme le rapporte la presse, a été arrêté alors qu'il regardait le dernier épisode à la télévision ${ }^{68}$. Comme le précise le magistrat Antonio Ingroia :

L'homogénéisation des cultures et des langues, favorisée par la télévision, frappe également l'univers mafieux. Bien loin d'être un symptôme du déclin fatidique de la mafia, c'est en revanche l'indice d'une nouvelle façon d'être des mafieux, de moins en moins différents des autres, de plus en plus intégrés dans la sociétée ${ }^{69}$.

L'intérêt des mafieux pour la représentation de leur univers et de leurs personnalités et la porosité entre les imaginaires relèvent donc d'une intégration des mafieux à la culture de masse.

Si la représentation du chef mafieux proposée dans la trilogie du Parrain a fortement structuré l'imaginaire collectif sur la mafia, alimentant les stéréotypes et le mythe qui l'entoure ${ }^{70}$, nous n'avons pas trouvé la trace d'une influence de ces films dans les témoignages de mafieux siciliens repentis. Il n'en va pas de même pour la Camorra puisque, comme le révèle Roberto Saviano dans Gomorra ${ }^{71}$, le boss Walter Schiavone s'est fait construire à Casal di Principe une villa identique à celle du personnage de Tony Montana interprété par Al Pacino dans Scarface de Brian De Palma (1983). Particulièrement perméable aux représentations cinématographiques, la Camorra est un cas à part puisque, en son sein, l'imitation de personnages mafieux devient une manière d'asseoir sa légitimité, comme si le chef pouvait récupérer le prestige de l'œuvre de fiction et s'inscrire dans un modèle vainqueur qui marque les esprits ${ }^{72}$. On remarque toutefois des constantes, quelle que soit l'œuvre cinématographique, notamment la persistance du luxe, en particulier des intérieurs. Dans Il capo dei capi, lorsque Riina va chez Stefano Bontate, il remarque une Ferrari qui trône dans la cour et des robinets en or $^{73}$. Il critique ce luxe, mais lorsqu'il fait construire sa villa à Corleone, dans le dernier épisode, il demande que tous les sols soient en marbre de Carrare. L'ostentation de richesse sert à asseoir le statut social du chef et ce stéréotype nourrit également l'imaginaire mafieux. De ce point de vue, la série Gomorra est exemplaire, car 
pour les scènes où apparaitt la maison de Pietro Savastano (saison 1), où les sanitaires sont en or massif, on a utilisé un bien immobilier confisqué à un boss de la Camorra ${ }^{74}$.

\section{Une représentation de la mafia sans mythification est-elle possible?}

Depuis sa diffusion en 2014 , la série Gomorra ${ }^{75}$ n'a pas manqué de soulever des polémiques médiatiques similaires à celles suscitées par Il capo dei capi. Leurs détracteurs les accusent de mythifier l'univers mafieux et de véhiculer une mauvaise image du territoire ainsi qu'un modèle dangereux pour les nouvelles générations ${ }^{76}$. Nous avons vu les aspects mythifiants et problématiques de Il capo dei capi, à savoir une représentation de la vie privée et familiale qui humanise le personnage de Riina, associée à une représentation de la violence somme toute feutrée. Le parti pris réaliste des scénaristes de Gomorra débouche en revanche sur une représentation crue du mal qui s'inscrit dans la même veine que The Wire, la série sur la criminalité à Baltimore, créée par David Simon et diffusée de 2002 à 2008. La noirceur des lieux, des intrigues et des personnages ultra violents rend toute la négativité de l'univers mafieux. Ainsi, le fait que deux productions cinématographiques si différentes essuient les mêmes critiques interroge : peut-on représenter la mafia sans la mythifier?

Comme nous l'avons dit, dès la diffusion des premiers épisodes du Capo dei capi, les polémiques éclatent, notamment suite à la condamnation du ministre de la Justice de l'époque. Les réalisateurs et les acteurs défendent la fiction, estimant qu'ils brisent l'omerta et dépassent les clichés du territoire dans une sorte de "catharsis collective " ${ }^{77}$. Pour Daniele Liotti, qui incarne Biagio Schirò à l'écran : «Il capo dei capi raconte la mafia du côté du mal mais le message qu'on laisse est clair: battons la mafia tous ensemble $\aleph^{78}$. Toutefois, force est de constater que ce message n'est pas passé de manière univoque : tandis que certains jeunes jouent à s'identifier aux mafieux après la diffusion des premiers épisodes la fiction ${ }^{79}$, d'autres à Corleone organisent une marche pour affirmer que « les Corléonais ne sont pas tous pareils $»^{80}$. Antonio Ingroia estime que toute représentation de la mafia est périlleuse et mythifiante :

Le spectateur moyen perçoit et apprécie le cinéma en tant que fiction, une fiction qui engendre des mythes et des événements romanesques qui, bien qu'inspirés de la réalité la plus crue, provoquent la fascination. C'est pour cette raison que [...] certaines représentations finissent par diffuser, au-delà des meilleures intentions de leurs auteurs, le charme négatif du héros du mal ${ }^{81}$.

Michele Serra, quant à lui, défend la qualité de la représentation et le choix d'une focalisation sur la figure de Riina car « le problème n'est pas de raconter le mal mais de ne pas l'avoir assez raconté » :

Qu'est-ce qui influence le plus les consciences, qu'est-ce qui change la culture et la mentalité des individus et des communautés, une rhétorique simpliste qui relègue le crime à sa seule horreur ou une définition véridique, difficile, intelligente de la puissance mafieuse ?82

Ces deux opinions montrent clairement la complexité de la question de la représentation de la mafia et de son impact sur les spectateurs. Au vu de l'âpreté et des similitudes des polémiques déclenchées par Il capo dei capi et plus tard par Gomorra, le problème semble apparemment insoluble car l'émulation et la mythification sont des risques inhérents à la représentation et à sa diffusion dans les médias. Toutefois, malgré les nombreuses différences entre les deux séries, l'irréalisme - dans le cas de Il 
capo dei capi - et l'inexistence - dans Gomorra - de l'antimafia (exception faite du magistrat qui apparaît sporadiquement dans la saison 4) semblent être de puissants vecteurs de mythification puisque le chef jouit d'une (relative) impunité qui forge son image d'invincible dans l'imaginaire collectif. On pourrait être tenté de penser qu'une représentation plus équilibrée des valeurs de l'État de droit face à celles de la criminalité serait la solution, puisque des séries comme La Piovra (dix saisons, entre 1984 et 2001) ou plus récemment Narcos (en partie consacrée à la lutte des agents de la DEA contre Pablo Escobar et le cartel de Medellin), construites sur le point de vue des enquêteurs, ont été globalement perçues comme salutaires pour déconstruire le mythe.

\section{BIBLIOGRAPHY}

Bolzoni, Attilio \& D’Avanzo, Giuseppe (2007), Il capo dei capi. Vita e carriera criminale di Totò Riina, Milan, Rizzoli, BUR (1 ${ }^{\text {ère }}$ éd : 1993).

Cianciabella, Elisa (2017), La rappresentazione televisiva della criminalità organizzata : gli effetti sull'opinione pubblica, mémoire de recherche, sous la direction de Nando dalla Chiesa, Université de Milan. Lien URL : https://www.stampoantimafioso.it/wp-content/uploads/2018/05/ TESI_CIANCIABELLA.pdf (consulté le 10 juillet 2019)

Ciancimino, Massimo \& La Licata, Francesco (2010), Don Vito. Le relazioni segrete fra Stato e mafia nel racconto di un testimone d'eccezione, Milan, Feltrinelli.

Fumarola, Silvia (2007), « Il capo dei capi un film che riscatta noi siciliani », La Repubblica, 23 octobre 2007. Lien URL : https://ricerca.repubblica.it/repubblica/archivio/repubblica/ 2007/10/23/il-capo-dei-capi-un-film-che.html?ref=search (consulté le 5 juillet 2019)

« Gomorra, "pizzo" per la casa del boss. Condannato il location manager » (2018), La Repubblica, 7 février 2018. Lien URL : https://napoli.repubblica.it/cronaca/2018/02/07/news/ gomorra_pizzo_per_la_casa_del_boss_condannato_il_location_manager-188279185/ (consulté le 9 juillet 2019)

Ingroia, Antonio (2007), « Raccontare la mafia senza il fascino del male », La Repubblica, 24 novembre 2007. Lien URL : https://ricerca.repubblica.it/repubblica/archivio/repubblica/ 2007/11/24/raccontare-la-mafia-senza-il-fascino-del.html?ref=search (consulté le 8 juillet 2019)

« La moglie di Riina all'attacco : danneggiata nella fiction » (2007), La Repubblica, $1^{\text {er }}$ décembre 2007. Lien URL : https://ricerca.repubblica.it/repubblica/archivio/repubblica/2007/12/01/lamoglie-di-riina-all-attacco-danneggiata.html?ref=search (consulté le 7 juillet 2019)

« La série Gomorra glorifie-t-elle la mafia? » (2019), Lien URL : https://www.huffingtonpost.fr/ entry/serie-gomorra-glorifie-t-elle-la-mafia_fr_5c9cccf1e4b072a7f60611a1 (consulté le 10 juillet 2019)

Mutolo, Gaspare \& Vinci, Anna (2013), La mafia non lascia tempo. Vivere, uccidere, morire dentro Cosa nostra. Il braccio destro di Totò Riina si racconta, Milan, Rizzoli.

Oliva, Ernesto \& Palazzolo, Salvo (2006), Bernardo Provenzano. Il ragioniere di Cosa nostra, Soveria Mannelli, Rubbettino. 
« Polemiche su serie tv su Totò Riina Capo dei capi » (2007), dépêche de l'agence de presse Ansa publiée sur le blog palermomania.it. Lien URL : https://www.palermomania.it/news/lifestylemondo/polemiche-su-serie-tv-su-toto-riina-capo-dei-capi-5619.html (consulté le 10 juillet 2019) Ravveduto, Marcello (2019), Lo spettacolo della mafia. Storia di un immaginario tra realtà e finzione, Turin, Gruppo Abele.

Saviano, Roberto (2006), Gomorra. Viaggio nell'impero economico e nel sogno di dominio della camorra, Milan, Mondadori.

Serra, Michele (2007), « Se Mastella vuole spegnere la fiction su Totò Riina », La Repubblica, 27 novembre 2007. Lien URL : https://ricerca.repubblica.it/repubblica/archivio/repubblica/ 2007/11/27/se-mastella-vuole-spegnere-la-fiction-su.html?ref=search (consulté le 11 juillet 2019)

« Totò Riina : "Il capo dei capi” fra realtà, critica e fantasia » 2009, 6 octobre 2009. Lien URL :

http://www.osservatoriesterni.it/schede/toto-riina-il-capo-dei-capi-fra-realta-critica-e-fantasia (consulté le 6 juillet 2019)

Viviano, Francesco \& Ziniti Alessandra (2012), Visti da vicino. Falcone e Borsellino gli uomini e gli eroi, Rome, Aliberti.

Torrealta, Maurizio (2010), La trattativa, Milan, Rizzoli.

Traversa, Fabio (2007), « Il capo dei capi distrae un latitante, arrestato dai carabinieri. Continuano le polemiche. Costanzo: "buona tv" ", La Repubblica, 30 novembre 2007. Lien URL : http://realityshow.blogosfere.it/post/88361/il-capo-dei-capi-distrae-un-latitante-arrestatodalla-polizia-continuano-le-polemiche-costanzo-buona (consulté le 9 juillet 2019)

Viviano, Francesco (2007), « Corleone, studenti in marcia : no alla mafia », La Repubblica, 28 novembre 2007. Lien URL : https://ricerca.repubblica.it/repubblica/archivio/repubblica/ 2007/11/28/corleone-studenti-in-marcia-no-alla-mafia.html?ref=search (consulté le 5 juillet 2019)

Ziniti, Alessandra (2007), « Fiction su Riina, è polemica. Propone modelli sbagliati », La Repubblica, 15 novembre 2007. Lien URL : https://ricerca.repubblica.it/repubblica/archivio/repubblica/ 2007/11/15/fiction-su-riina-polemica-propone-modelli-sbagliati.html?ref=search (consulté le 11 juillet 2019)

\section{Filmographie}

Les Affranchis (Goodfellas), scénario Nicholas Pilesti et Martin Scorsese, réalisation Martin Scorsese, 145', USA, 1990.

Il capo dei capi (6 épisodes), série écrite par Claudio Fava, Stefano Bises et Domenico Starnone, réalisation Enzo Monteleone et Alexis Sweet, production Taodue, Italie, 2007.

I cento passi, réalisation Marco Tullio Giordana, 114', Italie, 2000.

Il generale dalla Chiesa, série, réalisation Giorgio Capitani, 198', 2007.

Giovanni Falcone, scénario et réalisation Giuseppe Ferrara, 124', Italie, 1993.

Giovanni Falcone. L'uomo che sfidò Cosa nostra, par Antonio et Andrea Frazzi, Italie, 2006

Gomorra - La serie, série écrite par Giovanni Bianconi, Stefano Bises, Leonardo Fasoli, Ludovica Rampoldi, Roberto Saviano ; réalisation Stefano Sollima et Claudio Cupellini, Italie, 2014-2019.

Le Parrain (The Godfather), scénario Francis Ford Coppola et Mario Puzo, réalisation Francis Ford Coppola, 175', USA, 1972. 
La Piovra, dix mini-séries, Italie, 1984-2010.

Narcos, série écrite par Chris Brancato, Carlo Bernard et Doug Miro, réalisation José Padilha, USA, 2015-2017.

Paolo Borsellino, minisérie écrite par Giancarlo De Cataldo, Leonardo Fasoli et Mimmo Rafele, réalisation Gianluca Maria Tavarelli, 183', Italie, 2004.

Scarface, scénario Oliver Stone, réalisation Brian De Palma, 170', USA, 1983.

\section{NOTES}

1. En parallèle (1977-1983), les Corléonais se lancent dans une guerre ouverte contre l'État en assassinant des représentants politiques, des membres des forces de l'ordre, des magistrats et même le préfet de Palerme. Certains assassinats sont décidés par la Cupola (commission régionale, organe décisionnel de Cosa nostra, la mafia sicilienne) et exécutés par les hommes de Riina, mais il en décide d'autres de son propre chef.

2. Il est décédé en novembre 2017 après 24 ans passés en détention à l'isolement, selon les règles du régime carcéral 41 bis (cf. à ce propos l'article de Marcello Soddu dans ce $\mathrm{n}^{\circ} 36$ des Cahiers de Narratologie). Voir https://www.repubblica.it/cronaca/2017/11/17/news/ e_morto_il_boss_toto_riina_da_24_anni_era_al_41_bis-181309472/ (consulté le 18 juin 2019)

3. Bolzoni et D'Avanzo 2007, p. 8. La 1ère édition, remontant à 1993, est réédité en 2007, à l'occasion de la diffusion de la fiction à la télévision. Toutes les citations de cet article sont traduites par nos soins.

4. Série en 6 épisodes. Épisode 1: 1943-1962 ; épisode 2: 1963-1969; épisode 3 : 1969-1978; épisode 4 : 1978-1981 ; épisode $5: 1982-1992$; épisode $6: 1992-1993$.

5. Fils de Giuseppe Fava, journaliste assassiné par la mafia en janvier 1985 à Catane, Claudio Fava est un homme politique engagé à gauche, à l'époque membre du parti des Democratici di Sinistra.

6. Il capo dei capi, 2007, épisode 1.

7. Il capo dei capi, épisode 4.

8. Bolzoni et D'Avanzo 2007, p. 7.

9. En référence aux mafieux des cosche palermitaines.

10. Bolzoni et D'Avanzo 2007, p. 49-50.

11. Percepteur des impôts de Palerme, il faisait le lien avec les membres de la Démocratie Chrétienne proches de Cosa nostra comme Salvo Lima.

12. Jugement rendu en 1987. Il s'agit de la première victoire judiciaire de l'État italien contre Cosa nostra, dont les principaux chefs sont condamnés à la prison à vie.

13. Il capo dei capi, épisode 6.

14. Ibidem.

15. Gaspare Mutolo rencontre Riina en prison dans les années 1960 et devient l'un de ses plus fidèles soldats. Après son arrestation, Mutolo décide de collaborer avec la justice en 1992. Cf. Mutolo et Vinci 2013.

16. Il capo dei capi, épisode 4.

17. Riina s'adressant à Beppe, homme de confiance de Stefano Bontade (cosca de Santa Maria del Gesù). Il capo dei capi, épisode 4.

18. Il capo dei capi, épisode 4.

19. Pour le kidnapping du jeune Antonio Caruso, le massacre de Viale Lazio (exécution du boss Michele Cavataio commandité par Badalamenti dans la série, mais dans les faits le commanditaire est Bontate) et l'assassinat du procureur Scaglione (1971).

20. Il capo dei capi, épisode 2. 
21. Il capo dei capi, épisode 3.

22. Bolzoni - D'Avanzo 2007, p. 128. Scène représentée dans l'épisode 3.

23. Il capo dei capi, épisode 4.

24. Ibidem.

25. Il s'agit en fait de Luciano Raia, dont le nom a été changé dans la fiction. L'épisode est scrupuleusement repris de la biographie (voir Bolzoni - D’Avanzo 2007, p. 68-78). Déclaré fou, Luciano Raia est enfermé dans un asile, où il meurt quelques temps plus tard. Dans la série, le personnage de Maino se suicide après la fin du procès.

26. Il capo dei capi, épisode 2.

27. Cette activité criminelle, interdite en Sicile par les chefs mafieux pour ne pas attirer l'attention des forces de l'ordre, était en revanche pratiquée ailleurs, en particulier par la mafia calabraise, la 'Ndrangheta. Grâce aux enlèvements, les Corléonais amassent les fonds nécessaires pour investir dans le trafic de drogue et le secteur de la construction.

28. Ce déséquilibre ne reflète pas la réalité. Il est plus juste de parler d'un duumvirat (cf. Oliva et Palazzolo 2006).

29. Signifiant «le massacre ", ce terme faisant référence à la tradition de la pêche aux thons est utilisé pour évoquer la seconde guerre de mafia, qui a conduit à l'élimination des familles palermitaines sans que celles-ci ne réussissent à répliquer.

30. Il capo dei capi, épisode 4 (dernière scène).

31. Il capo dei capi, épisode 5.

32. Il capo dei capi, épisode 5.

33. Provenzano dit à Riina, à propos des référents politiques : «Peut-être que tu as eu tort de leur faire confiance. Moi je n'ai jamais eu confiance en certaines personnes. Ceux qui ont le cul bien au chaud à Rome, qu'est-ce qu'ils en ont à foutre de nos procès? C'était mieux quand on réglait nos problèmes tout seuls ", Il capo dei capi, épisode 6.

34. Forme sicilienne de zio (oncle), cet appellatif est une marque de respect et précède souvent le prénom ou diminutif du chef mafieux.

35. Il capo dei capi, épisode 6.

36. Lorsque Giovanni Brusca - exécutant de l'attentat - lui demande ce qu'en pense Provenzano, Riina répond : «Binnu est toujours d'accord avec moi », Il capo dei capi, épisode 6.

37. La famille de Provenzano revient s'installer à Corleone en avril 1992, un mois avant le massacre de Capaci, ce qui témoigne d'une rupture en interne.

38. Cf. Torrealta 2010 ainsi que Ciancimino - La Licata 2010.

39. Il capo dei capi, épisode 6.

40. Ces deux policiers ont mené les enquêtes avec les juges Falcone et Borsellino. Ils sont assassinés à une semaine d'intervalle en 1985. Voir Il capo dei capi, épisode 5.

41. Ces entrevues ont lieu dans l'ascenseur ou hors du Palais de Justice car Schirò est convaincu qu'il y a une taupe mais le procureur sous-entend qu'il est paranoïaque.

42. Le procureur Costa vient d'être assassiné, après avoir signé seul des mandats d'arrêt (1980).

43. Il capo dei capi, épisode 4.

44. Les références sont nombreuses, mais voir par exemple Viviano et Ziniti 2012.

45. Il capo dei capi, épisode 4.

46. Il capo dei capi, épisode 5.

47. Il capo dei capi, épisode 6.

48. Il capo dei capi, épisode 3.

49. Il capo dei capi, épisode 3.

50. Fumarola 2007.

51. Riina et Provenzano étaient surnommés le belve, les bêtes féroces, par les autres chefs mafieux car ils tuaient à tour de bras.

52. Il capo dei capi, épisode 5. 
53. Il capo dei capi, épisode 5.

54. Fumarola 2007.

55. Giovanni Falcone, de Giuseppe Ferrara, 1993 ; I cento passi, de Marco Tullio Giordana, 2000.

56. Paolo Borsellino, de Gianluca Maria Tavarelli, 2004 ; Giovanni Falcone. L'uomo che sfidò Cosa nostra, d'Antonio et Andrea Frazzi, 2006 ; Il generale dalla Chiesa, de Giorgio Capitani, 2007.

57. « Fiction su Riina, Grasso : “A scuola se ne discuta” » 2007.

58. Palazzolo 2007.

59. « Oltre dieci milioni per Benigni. Quasi otto per Il capo dei capi » 2007.

60. https://www.rosalio.it/2007/11/30/il-capo-dei-capi-pro-o-contro/

61. La veuve de ce policier, assassiné le 21 juillet 1979, a violemment critiqué la série, estimant que son mari "n'avait pas besoin d'un inexistant Schirò qui le pousse à combattre la mafia " (« Totò Riina : "Il capo dei capi” fra realtà, critica e fantasia » 2009).

62. Dans l'épisode 3, la scène du mariage de Totò Riina évoque la scène d'ouverture du Parrain. Dans l'épisode 4, la scène où Luchino Bagarella rit pendant que Totò Riina parle et ce dernier fait semblant de s'énerver pour lui faire peur, rappelle celle des Affranchis où Joe Pesci fait semblant de s'énerver contre Ray Liotta.

63. Les épisodes complets, disponibles sur YouTube, ont entre 177000 et 475000 vues.

64. Palazzolo 2007.

65. Ibidem.

66. Ibidem.

67. «La moglie di Riina all'attacco: danneggiata nella fiction» 2007. La plainte porte sur un montage parallèle de l'épisode 3 montrant, d'une part, Ninetta se défendant au tribunal dans le cadre d'une procédure d'assignation à résidence loin de Corleone et d'autre part, l'enlèvement de la femme et du fils de Schirò, dont la libération est conditionnée à la décision du tribunal. Le préjudice d'atteinte à l'image dénoncé a fait l'objet d'une demande de compensation mais nous n'avons pu trouver les suites judiciaires de la procédure.

68. Cf. Traversa 2007.

69. Ingroia 2007.

70. Cf. Ravveduto 2019.

71. Saviano 2006. Voir en particulier le chapitre « Hollywood", consacré aux connexions et aux contaminations entre les imaginaires mafieux réels et leurs représentations dans le cinéma, majoritairement américain.

72. L'instabilité de la Camorra, due à sa structure horizontale, est certainement l'une des clés permettant de comprendre cette urgence de vouloir s'imposer au niveau des apparences et l'ostentation de la richesse, en utilisant les représentations cinématographiques pour nourrir son imaginaire.

73. Il capo dei capi, épisode 4.

74. Avant que ce bien ne soit confisqué, le manager de la production a payé le pizzo au clan de Torre Annunziata pour tournage les premiers épisodes (cf. «Gomorra, "pizzo" per la casa del boss. Condannato il location manager » 2018).

75. Avec près de 700000 téléspectateurs pour chaque épisode de la première saison, elle bat des records d'audience malgré sa diffusion sur une chaîne payante (Sky TV). La version française sur Canal+ rassemble également près d'un million de téléspectateurs et on compte 130 millions de fans à travers le monde. Voir https://www.lastampa.it/cronaca/2014/07/17/news/la-serie-tvgomorra-pagava-il-pizzo-1.35734342 et https://www.huffingtonpost.fr/entry/serie-gomorraglorifie-t-elle-la-mafia_fr_5c9cccf1e4b072a7f60611a1 (consultés le 10 juillet 2019).

76. Cf. «Polemiche su serie tv su Totò Riina Capo dei capi» 2007. Pour l'analyse détaillée des polémiques médiatiques sur les représentations de la criminalité organisée et plus particulièrement sur la série Gomorra, voir Cianciabella 2017.

77. Fumarola 2007.

Cahiers de Narratologie, 36 | 2019 
78. Viviano 2007.

79. «Au bar A Maronnuzza, depuis quelques jours les jeunes s’appellent "Binnu”, “Totò u curtu”, "Lucianeddu". Il y en a même un qui boîte en s'appuyant sur un bâton pour imiter la démarche de Luciano Leggio », Ziniti 2007.

80. Viviano 2007.

81. Ingroia 2007.

82. Serra 2007

\section{ABSTRACTS}

La fiction Il capo dei capi réalisée en 2007 est une représentation de Totò Riina qui fait débat. Il convient dès lors de s'interroger sur les ressorts de cette représentation car l'image du personnage forge également sa mémoire auprès du public, a fortiori dans le cas d'une série télévisée qui, comme Il Capo dei capi, rencontre un large succès y compris à l'étranger. Ce pouvoir de façonner la mémoire dans l'imaginaire collectif nous impose d'étudier non seulement la représentation en soi et le message qu'elle fait passer mais également la réception de la série lors de sa diffusion. Cet article analyse donc la sédimentation des différentes facettes du personnage de Totò Riina afin de comprendre pourquoi il s'agit d'une représentation problématique et mythifiante dont le succès a déclenché de vives polémiques.

La fiction Il capo dei capi realizzata nel 2007 è una rappresentazione di Totò Riina che fa discutere. Bisogna quindi interrogarsi sulle peculiarità di tale rappresentazione perché l'immagine del personaggio costruisce anche la sua memoria presso il pubblico, a maggior ragione nel caso di una serie televisiva come Il Capo dei capi, che ha avuto un grande successo anche all'estero. Questo potere di dar forma alla memoria nell'immaginario collettivo impone di studiare non solo la rappresentazione in sé e il messaggio che essa veicola, ma anche la ricezione della serie al momento della diffusione. L'articolo esamina la sedimentazione delle varie sfaccettature del personaggio di Riina per capire perché ne è stata data una rappresentazione problematica e mitizzante, il cui successo ha scatenato aspre polemiche.

\section{INDEX}

Mots-clés: mafia, représentation, Totò Riina, fiction télévisée, Capo dei capis

\section{AUTHOR}

\section{CHARLOTTE MOGE}

Charlotte Moge, agrégée d'italien et docteure en histoire contemporaine, est maître de conférences en Études italiennes à l'Université Jean Moulin Lyon 3 et membre de l'UMR Triangle 5206. Ses recherches portent sur le mouvement antimafia, la mémoire des victimes de la lutte contre la mafia et sur la criminalité mafieuse en Italie. Parmi ses publications récentes : C. Moge, G. Panvini \& P. Picco (dir.), « Sans recourir à la violence » : la société italienne face aux 
terrorismes et aux mafias, Laboratoire italien (2019) ; « Eroe, uomo, santo ? Il paradosso della memoria di Giovanni Falcone » in T. Caliò \& L. Ceci (dir.), L'immaginario devoto tra mafie e antimafia. Riti, culti e santi (2017); « La mobilisation antimafia de 1992 », Rivista di Studi e ricerche sulla criminalità organizzata (2016) ; « Parler de mafia. La classe politique face à la violence mafieuse de 1963 à 1992 », in R. Descendre \& J.-L. Fournel (dir.), Langages, politique, histoire avec Jean-Claude Zancarini (2015). 